\title{
Own Vibrations of Bodies Interacting with Unlimited Deformable Environment
}

\author{
Ismail Ibrahimovich Safarov', Muhsin Khudoyberdiyevich Teshaev², \\ Zafar Ixtiyorovich Boltayev² \\ ${ }^{1}$ Tashkent Chemistry, Technological Institute, Tashkent, Republic of Uzbekistan \\ ${ }^{2}$ Bukhara Technological, Institute of Engineering, Bukhara, Republic of Uzbekistan \\ Email: safarov54@mail.ru
}

How to cite this paper: Safarov, I.I., Teshaev, M.K. and Boltayev, Z.I. (2018) Own Vibrations of Bodies Interacting with Unlimited Deformable Environment. Open Access Library Journal, 5: e4432. https://doi.org/10.4236/oalib.1104432

Received: February 22, 2018

Accepted: March 26, 2018

Published: March 29, 2018

Copyright $\odot 2018$ by authors and Open Access Library Inc.

This work is licensed under the Creative Commons Attribution International License (CC BY 4.0).

http://creativecommons.org/licenses/by/4.0/

\begin{abstract}
The vibrations of deformed bodies interacting with an elastic medium are considered. The problem reduces to finding those values of complex Eigen frequencies for which the system of equations of motion and the radiation conditions have a nonzero solution to the class of infinitely differentiable functions. It is shown that the problem has a discrete spectrum located on the lower complex plane and the symmetric spectrum is an imaginary axis.
\end{abstract}

\section{Subject Areas \\ Continuum Mechanics}

\section{Keywords}

Damping Coefficient, Natural Frequencies, Elastic Medium, Shells, Oscillations

\section{Introduction}

In this paper we consider oscillations of cylindrical bodies in a deformable medium [1] [2] [3]. From the physical point of view, the damping in an ideal elastic medium is explained by the radiation of energy excited by the natural oscillations due to divergent elastic waves. The behavior of complex Eigen frequencies depending on the geometric and physic mechanical parameters of the system is investigated. The environment of cylindrical and spherical bodies is considered as elastic, viscoelastic and multicomponent. The obtained numerous results are compared on a computer. A piecewise homogeneous mechanical system is regarded as dissipative homogeneous and inhomogeneous. The ideal elastic body 
has no losses [4] [5]. Such a body is characterized by a linear single-valued relationship between stress and strain throughout the entire period of the alternating voltage. Hence it follows that stress and deformation are always in phase. The energy dissipation of an elastic wave will occur if the stress and strain are not connected by an unambiguous dependence during the period of oscillations. The absence of such an unambiguous relationship between stress and deformation arises when temporal derivatives appear in the equation connecting them. Even if the equation is linear with respect to stress and strain, the presence of time derivatives is always associated with dissipation. As a result, with an alternating voltage there is a hysteresis effect. This means that in the frequency range in which attenuation has an appreciable magnitude, the strain will lag behind the voltage. The presence of only a nonlinear connection between stress and deformation (without time derivatives in the equation) has two effects. Such a connection, firstly, leads to the interaction of the elastic wave under consideration with other waves (for example, with thermal vibrations) and as a result there is a redistribution of energy between the waves. Secondly, the considered wave will generate higher harmonics, transferring their energy to them. In both cases, the interaction depends on the strain amplitude. The nonlinear relationship between stress and strain in the presence of time derivatives also leads to damping, which depends on the strain amplitude. The Eigen vibrations of the rods and shells in an elastic medium are considered in [6] [7] [8]. In these works, the environment of rods and shells is replaced by elastic springs, i.e. the coefficient of spring stiffness is taken into account in the calculation. In [9], free oscillations of spherical shells interacting with an elastic medium. Numerical results are obtained and analysis is made. In the present paper, in contrast to the known papers, instead of the Somerfield radiation condition at infinity, an alternative condition is considered-non-reflecting boundaries.

\section{The Body's Own Oscillations in the Medium}

Three problems of natural oscillations of bodies interacting with the medium are considered.

We consider model problems for the wave equation and, for example, demonstrate the general scheme for constructing a solution with allowance for the radiation principle. The solution of the problem of natural oscillations of a semi-infinite rod with mass $m$ (Figure 1 ) has the form

$$
u(x, t)=\left\{\begin{array}{lc}
W(x) \mathrm{e}^{-i \omega t}, & 0<x<x_{1} \\
a \mathrm{e}^{i(k x-\omega t)}, & x_{1}>1
\end{array}\right.
$$

where $\omega=\omega_{R}+i \omega_{I} ; \omega_{I}>0$. Let $\omega / k=c-$ known real speed, $\omega / c=k-$ complex wave number. On an infinite section

$$
u(x, t)=a \mathrm{e}^{-i \omega t} \mathrm{e}^{-i \omega x / c} .
$$

We define the dependence of $u$ on $x$ for $t=0$ : 


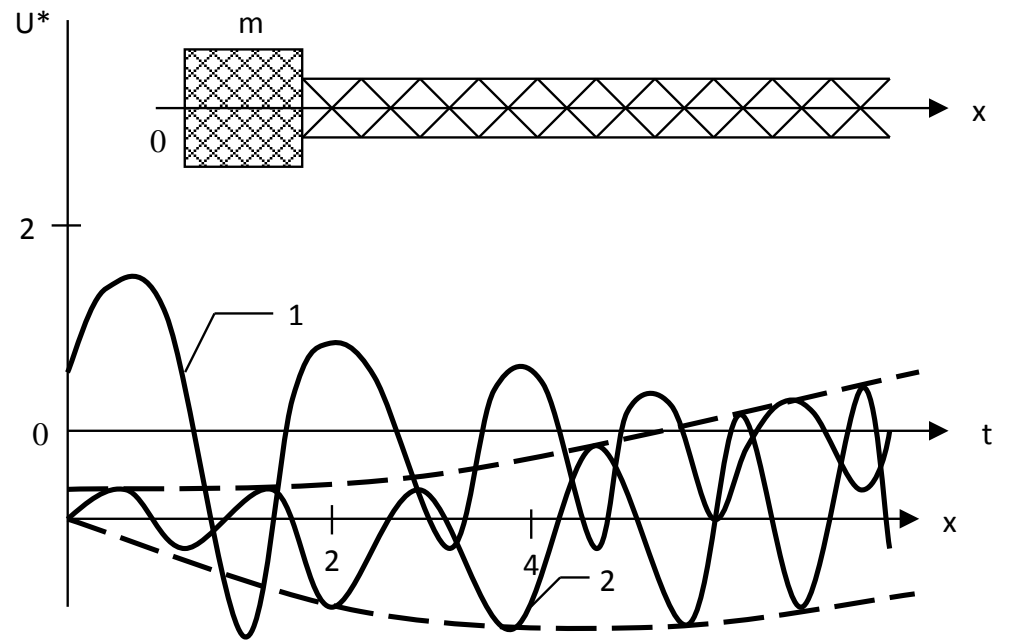

Figure 1. Calculation scheme.

$$
u(x, 0)=a \mathrm{e}^{\frac{i}{c}\left(\omega_{R}-i \omega_{I}\right) x}=a \mathrm{e}^{\frac{\omega_{I}}{c} x} \mathrm{e}^{\frac{i}{c} \omega_{R} x} .
$$

As can be seen, Figure 1 with increasing $x$ displacement $u(x, 0)$ at the expense of $\mathrm{e}^{\frac{\omega_{I}}{c} x}$ term increases to infinity. Thus, when solving the problems of natural oscillations of cylindrical bodies in an elastic medium at infinity $(r \rightarrow \infty)$ the potential of the displaced Summerfield is not fulfilled. Thus, a new type of condition is required when $r \rightarrow \infty$.

\subsection{Consider the Natural Oscillations of the Composite Rod, to the Left It Is Fixed, and to the Right Is the Damper (Figure 1)}

The main goal in this problem is to show the independence of the Eigen frequencies of the left rod from the length of the right rod if shortened Summerfield conditions are put at the end of the right rod [10] [11].

It is required to find the solution of the following homogeneous equation:

$$
G \frac{\partial^{2} u}{\partial x^{2}}-\rho \frac{\partial^{2} u}{\partial t^{2}}=0,
$$

$G=E F$, Young's $E$-module, $F$-cross-sectional area, $\rho$-density of the rod material) with the following boundary conditions

$$
\begin{aligned}
& U(0, t)=0 ; \\
& U\left(x_{1}-0, t\right)-U\left(x_{1}+0, t\right)=0 ; \\
& G_{1} U_{x}^{1}\left(x_{1}-0, t\right)-G_{2} U_{x}^{1}\left(x_{1}+0, t\right)=0, \\
& G_{2} U_{x}^{1}\left(x_{2}, t\right)+\mu U_{t}^{1}\left(x_{2}, t\right)=0 .
\end{aligned}
$$

We express the solution of (2.1) in the form

$$
U(x, t)=U(x) \mathrm{e}^{-i w t}
$$

where $U(x)$-amplitude function, for each section $0<x_{1}<x$ and $0<x_{1}<x$; $x_{1}<x<x_{2}$, we write in the form 


$$
U(x)= \begin{cases}C_{1} \mathrm{e}^{i \frac{\omega}{C_{p 1}}}+C_{2} \mathrm{e}^{-i \frac{\omega}{C_{p 1}} x}, & 0<x<x_{1} \\ C_{1}^{1} \mathrm{e}^{i \frac{\omega}{C_{p 2}} x}+C_{2}^{1} \mathrm{e}^{-i \frac{\omega}{C_{p 2}} x}, & x_{1}<x<\infty\end{cases}
$$

To determine the constants $C$ we have the following boundary conditions:

$$
\begin{aligned}
& U(0)=0 ; \\
& U\left(x_{1}-0\right)-U\left(x_{1}+0\right)=0 ; \\
& G_{1}\left(x_{1}-0\right)-G_{2} U^{1}\left(x_{1}+0\right)=0, \\
& G_{2} U^{1}\left(x_{2}\right)-\operatorname{l\omega } \mu U\left(x_{2}\right)=0 .
\end{aligned}
$$

From the boundary conditions we obtain

$$
\begin{aligned}
& {\left[\frac{G_{1}}{C_{p 1}}\left(1+\beta_{1} \mathrm{e}^{-\frac{2 i \omega}{C_{p 2}} x_{1}} \mathrm{e}^{-\frac{2 i \omega}{C_{p 2}} x_{2}}\right)-\frac{G_{2}}{C_{p 2}}\left(1-\beta_{1} \mathrm{e}^{-\frac{2 i \omega}{C_{p 2}} x_{1}} \mathrm{e}^{-\frac{2 i \omega}{C_{p 2}} x_{2}}\right)\right] \mathrm{e}^{\frac{2 i \omega}{p_{1} 1} x_{1}}} \\
& +\frac{G_{2}}{C_{p 2}}\left(1+\beta_{1} \mathrm{e}^{-\frac{2 i \omega}{C_{p 2}} x_{1}} \mathrm{e}^{\frac{2 i \omega}{C_{p 2}} x_{2}}\right)-\frac{G_{1}}{C_{p 1}}\left(1-\beta_{1} \mathrm{e}^{-\frac{2 i \omega}{C_{p 2}} x_{1}} \mathrm{e}^{-\frac{2 i \omega}{C_{p 2}} x_{2}}\right)=0
\end{aligned}
$$

where $\beta=\left\{\frac{G_{2}}{C_{p 2}}-\mu\right\} /\left\{\frac{G_{2}}{C_{p 2}}+\mu\right\}$. If we set $\mu=G_{2} / C_{p 2}$, that is, the right-hand end is set to the radiation conditions $\beta_{1}=0$, then (2.6) takes the following form

$$
\left\{\frac{G_{2}}{C_{p 2}}-\frac{G_{2}}{C_{p 2}}\right\} \mathrm{e}^{\frac{2 i \omega}{C_{p 1}} x_{1}}+\left\{\frac{G_{2}}{C_{p 2}}+\frac{G_{1}}{C_{p 1}}\right\}=0
$$

The spectrum of eigenvalues is defined by formula

$$
\begin{gathered}
\omega_{k}=\frac{C_{p 1} k \pi}{x_{1}}-i \frac{C_{p 1}}{2 x_{1}} \ln \frac{G_{1} C_{p 1}+G_{2} C_{p 2}}{G_{1} C_{p 1}-G_{2} C_{p 2}} \text { at } G_{2} C_{p 1}>G_{1} C_{p 2} \quad(k=0,1,2, \cdots) \\
\omega_{k}=\frac{C_{p 1}(2 k+1) \pi}{2 x_{1}}-i \frac{C_{p 1}}{2 x_{1}} \ln \frac{G_{1} C_{p 2}+G_{2} C_{p 1}}{G_{2} C_{p 1}-G_{2} C_{p 1}} \text { at } G_{2} C_{p 1}<G_{1} C_{p 2} .
\end{gathered}
$$

where by logarithm is meant the main real branch is the invalid part of the eigenvalues $\omega_{k}$ has the meaning of the frequency of natural oscillations. They exactly coincide with the natural frequencies of the left side of the rod $\left(0<x<x_{1}\right)$ with a fixed end at $G_{1} C_{p 2}>G_{2} C_{p 1}$.

When $G_{1} C_{p 2}<G_{2} C_{p 1}$ actual parts $u_{k}$ coincide with the natural frequencies of the rod, with a free right end. The imaginary parts $u_{k}$ have the meaning of the damping coefficients and are the same for all eigenvalues $u_{k}$. The logarithmic damping decrement decreases inversely proportional to the Eigen frequency number. If $G_{1}=G_{2}$ and $\tilde{\mu}_{1}=\tilde{\mu}_{2}$, then we get the natural oscillations of the rod, the left end is fixed, and the right damper. The frequency Equation (2.8) takes the following form

$$
\left\{\frac{G_{1}}{C_{p 1}}+\mu\right\}\left\{\frac{G_{1}}{C_{p 1}}-\mu\right\} e^{\frac{2 i \omega}{C_{p 1}} x_{i}}=0
$$


Natural frequency

$$
\omega_{k}=\frac{c_{p 1}^{(2 k+1)}}{2 x_{1}}-i \frac{c_{p 1}}{2 x_{1}} \ln \beta_{1} \quad(k=0,1,2, \cdots)
$$

Reflection is absent, since the supplied conditions are satisfied by a divergent cylindrical and spherical waves.

$$
\beta_{1}=\left\{\frac{G_{2}}{C_{\rho 2}}+\mu\right\} /\left\{\frac{G_{2}}{C_{\rho 2}}-\mu\right\},
$$

at $i=0$, there exists a real natural frequency. Real parts $\omega_{k}$ coincide with the eigen frequencies of the rod with free right the end. The imaginary parts are equal to zero. When $\mu=G_{1} / C_{p 1}$ semi-infinite rod, there is no natural frequency. In this way ( $\mu=G_{1} / C_{p 1}$ non-reflection conditions), the frequency Equations (2.6) do not depend on the length of the right rod.

\subsection{Anti-Flat Oscillations of a Cylinder Immersed in an Infinite Medium}

It can be shown that, for $R \gg 1$, the problem under consideration is equivalent to the problem of the eigen vibrations of a two-layered cylinder depicted in Figure 2, which is satisfied by the following equations of motion and boundary conditions [12]:

$$
\begin{aligned}
& \frac{\partial^{2} U}{\partial r^{2}}+\frac{1}{r} \frac{\partial U}{\partial r}-\frac{1}{C_{p k}^{2}} \frac{\partial^{2} U}{\partial t^{2}}=0, \quad k=1,2 \\
& U=0, r=r_{1} \\
& U(r-0)=U(r+0), \sigma_{r x_{3}}(r-0)=\sigma_{r x_{3}}(r+0), r=r_{2} \\
& \sigma_{r x_{3}}=\mu_{2} \frac{\partial u}{\partial r}, r=R
\end{aligned}
$$

where $\mu_{2}$-he Lame coefficient.

Now we determine the conditions for the absence of reflection for $r=R$. For $R \gg 1$ divergent waves has the form

$$
U(r, t)=\frac{1}{\sqrt{\alpha r}} \mathrm{e}^{i\left(\alpha r-\frac{\pi}{4}-\omega t\right)} .
$$

Since the asymptotic are valid

$$
H_{0}^{(1)}(\alpha r) \cong \sqrt{\frac{2}{\pi \alpha r}} \mathrm{e}^{i\left(\alpha r-\frac{\pi}{4}\right)},
$$

where $\alpha$-wave number; $y$ is the natural frequency. From relation (2.13) we have

$$
\frac{\partial U}{\partial r}=\frac{i \alpha}{\sqrt{\alpha r}} \mathrm{e}^{i\left(\alpha r-\frac{\pi}{2}-\omega t\right)}-\frac{\alpha}{2(\alpha r)^{2 / 3}} e^{i\left(\alpha r-\frac{\pi}{4}-\omega t\right)},
$$

or

$$
\frac{\partial U}{\partial r}=i \alpha u-\frac{1}{2 r} u
$$




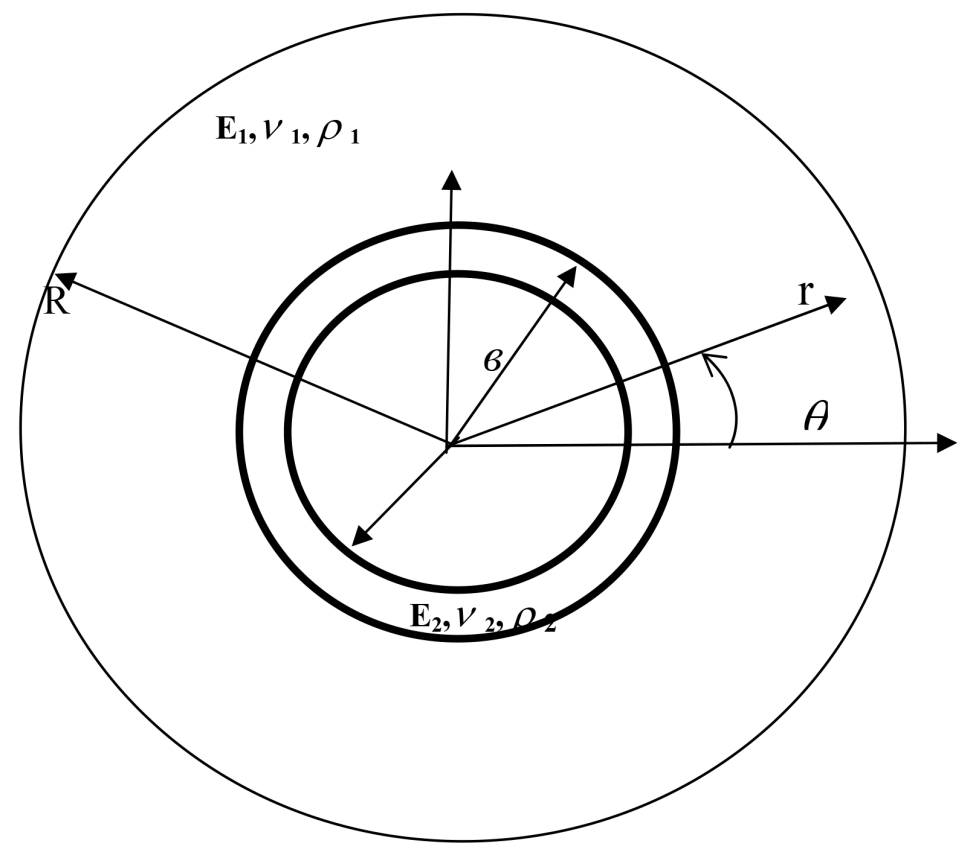

Figure 2. Calculation scheme.

Further

$$
\frac{\partial U}{\partial t}=-\frac{i \omega}{\sqrt{\alpha r}} \mathrm{e}^{i\left(\alpha r-\frac{\pi}{4}-\omega t\right)}
$$

or $\frac{\partial U}{\partial t}=-i \omega u$. After some transformations, we get

$$
\frac{\partial U}{\partial r}=-\frac{\alpha}{\omega} \frac{\partial U}{\partial t}-\frac{1}{2 r} U
$$

Thus, the condition for $r=R$ has the following form

$$
\begin{aligned}
& \left.\frac{\partial U}{\partial r}\right|_{r=R}=\left.\left(-\frac{\alpha}{\omega} \frac{\partial U}{\partial t}-\frac{1}{2 R} U\right)\right|_{r=R} \\
& \left.\sigma_{r x_{3}}\right|_{r=R}=\left.\frac{\mu_{2}}{C_{p 2}} \frac{\partial U}{\partial t}\right|_{r=R}-\left.\frac{\mu_{2}}{2 R} U\right|_{r=R}
\end{aligned}
$$

For radial oscillations, it follows directly from (2.17) that

$$
\left.\sigma_{r r}\right|_{r=R}=-\left.\frac{\mu_{2}}{C_{p 2}} \frac{\partial u}{\partial t}\right|_{r=R}-\left.\frac{\mu_{2}}{2 R} u\right|_{r=R}
$$

Under this condition (2.18), obtaining the frequency equation does not depend on the radius of the outer cylinder.

\subsection{Radial Oscillation of a Spherical Body}

Let us consider in an unbounded medium the radial oscillations of the cavity, accompanied by the emission of longitudinal sound waves, which leads to a loss of energy, and thus to a damping of the oscillations. When $C_{p} \gg C_{s}$ the prob- 
lem under consideration is equivalent to the problem of the natural oscillations of the spherical hole shown in Figure 2. The solution of this problem will be sought in the form of a "potential" of displacement $\varphi(r, t)$, satisfying equation

$$
\frac{\partial^{2} \varphi}{\partial r^{2}}+\frac{2}{r} \frac{\partial \varphi}{\partial r}-\frac{1}{C_{p 2}} \frac{\partial^{2} \varphi}{\partial t^{2}}=0
$$

The solution (2.19) presented in the form

$$
\varphi(r, t)=\left[\left(\frac{A}{r} \mathrm{e}^{i \alpha r e}+\frac{R}{r} \mathrm{e}^{-i a r}\right) \mathrm{e}^{-i \omega t}\right]
$$

satisfies the boundary conditions

$$
\left.\sigma_{r r}\right|_{r=a}=0
$$

and

$$
\left.\frac{\partial \varphi}{\partial r}\right|_{v=b}=\left.\left(-\frac{\alpha_{p}}{\omega} \frac{\partial u}{\partial t}-\frac{1}{r}\right)\right|_{r=b},
$$

where

$$
\begin{gathered}
\sigma_{r r}=-\mu\left[a_{t}^{2} \varphi+\frac{4}{r} \frac{\partial \varphi}{\partial r}\right], \\
a_{p}^{2}=\frac{\omega^{2}}{C_{p}^{2}} ; a_{t}^{2}=\frac{\omega^{2}}{C_{s}^{2}} ; C_{p}^{2}=\frac{\lambda+2 \mu}{\rho}-\text { longitudinal wave velocity; } C_{s}^{2}=\frac{\mu}{\rho}-
\end{gathered}
$$

velocity of propagation of transverse waves. Substituting (2.20) into (2.21), we obtain

$$
\varphi(r, t)=\frac{A}{r} \mathrm{e}^{i\left(a_{p} r-\omega t\right)}
$$

Substituting (2.21) into the boundary condition (2.21), we have

$$
\left(\kappa a \frac{C_{p}}{C_{s}}\right)^{2}=4(1-i \kappa a)
$$

from here at $C_{p} \gg C_{s}$

$$
\omega=\frac{2 C_{s}}{a}\left(1-i \frac{C_{s}}{C_{p}}\right)
$$

At $a \rightarrow \infty$ a natural frequencies $\omega \rightarrow 0$; When $C_{p} \rightarrow \infty$ the natural frequency takes the following form $\omega=\frac{2 C_{s}}{a}$. It is clear from (2.22) that $\omega=\omega_{R}+i \omega_{I}$ where $\omega_{R}=\frac{2 C_{s}}{a} ; \omega_{I}=\frac{C_{s}}{C_{P}} \ll 1$. The resulting expression (2.22) completely corresponds to the results of the work of Landau and Lifshitz, which are obtained on the basis of the Somerfield radiation condition.

Thus, two problems for an infinite and finite domain are equivalent. This is explained by the fact that at the outer boundary conditions are set for the absence of reflection, which in the general case are written in the form 


$$
L \boldsymbol{U}\left(R_{\infty}\right)=l \boldsymbol{U}\left(R_{\infty}\right)
$$

Here $L$ and $I$ linear differential operators, $R_{\infty}$-border selected area of infinite media, $\boldsymbol{U}$-vector of displacement. Equation (2.23) replaces the Somerfield radiation condition.

Equations (2.7), (2.18), and (2.21) is a particular case of (2.23). Equation (2.23) is the nonreflecting condition of the "Troyanovski - Safarov". In a particular case, it results in a shortening of the Sommerfeld radiation condition [13]

$$
\lim _{r \rightarrow \infty} \sqrt{r}\left(\frac{\partial \boldsymbol{U}}{\partial r}+i K_{1} \boldsymbol{U}\right)=0 .
$$

\section{The Eigen Vibrations of Piecewise-Homogeneous Cylindrical Systems}

We consider the natural oscillations of piecewise-homogeneous cylindrical bodies in an infinitely elastic medium (Figure 2). The purpose of which is to show the influence of piecewise homogeneity on natural frequencies and damping indices of a mechanical system.

The linear equation of motion in displacement potentials in the absence of volume forces has the form

$$
\begin{aligned}
& \Delta \phi_{k}-\frac{1}{c_{p j}^{2}} \frac{\partial^{2} \phi_{k}}{\partial t^{2}}=0 ; \Delta \psi_{z k}-\frac{1}{c_{s j}^{2}} \frac{\partial^{2} \psi_{z k}}{\partial t^{2}}=0 ; \\
& \Delta=\frac{1}{r} \frac{\partial}{\partial r}+\frac{1}{r^{2}} \frac{\partial^{2}}{\partial \theta^{2}}+\frac{\partial^{2}}{\partial r^{2}} ; \\
& u_{r k}=\frac{\partial \phi_{k}}{\partial r}+\frac{1}{r} \frac{\partial \psi_{z k}}{\partial \theta} ; u_{\theta k}=\frac{1}{r} \frac{\partial \phi_{k}}{\partial \theta}-\frac{\partial \psi_{z k}}{\partial r} ;
\end{aligned}
$$

Solutions of Equations (3.1) are sought in the form:

$$
\left.\begin{array}{l}
\phi_{k}(r, \theta, t)=\sum_{n=0}^{\infty} \varphi_{1 n}\left(\alpha_{k} r\right)\left\{\begin{array}{l}
\cos n \theta \\
-\sin n \theta
\end{array}\right\} \mathrm{e}^{-i \omega t} ; \\
\psi_{z k}(r, \theta, t)=\sum_{n=0}^{\infty} \varphi_{2 n}\left(\beta_{k} r\right)\left\{\begin{array}{c}
\sin n \theta \\
\cos n \theta
\end{array}\right\} \mathrm{e}^{-i \omega t} ;
\end{array}\right\}
$$

where $n$-integer; $\omega$-complex natural frequency; $r=\frac{r_{1}}{a_{0}}$. At infinity $(r \rightarrow \infty)$ The Sommerfeld conditions for each component are formulated. Substituting (3.2) into (3.1), we obtain the following ordinary differential equations:

$$
\nabla^{2} \varphi_{\text {in }}+K_{i}^{2} \varphi_{\text {in }}=0, K_{i}^{2}=\frac{\omega^{2}}{c_{i}^{2}}, i=1,2 ; c_{1}^{2}=\left(\lambda_{c}+2 \mu_{c}\right) / \rho_{c}, c_{2}^{2}=\mu_{c} / \rho_{c} .
$$

Let us consider the natural oscillations of a cylindrical hole in an elastic medium. On the boundary $r=a$ we set the condition free of stress, i.e.

$$
\left.\sigma_{r r}\right|_{r=a}=\left.\sigma_{r \theta}\right|_{r=a}=0 .
$$

Substituting (3.2) into (3.3), we obtain the frequency equation

$$
Z_{1 n} X_{2 n}+Z_{2 n} X_{1 n}=0
$$


where

$$
\begin{gathered}
X_{1 n}=\Omega_{0} H_{n+1}^{(1)}\left(\Omega_{0}\right)+\left(a_{n 2}^{1}-d_{1} \Omega_{0}^{2}\right) H_{0}^{(1)}\left(\Omega_{0}\right) ; \\
X_{2 n}=n\left[(n-1) H_{n}^{(1)}\left(\Omega_{1}\right)-\Omega_{1} H_{n+1}^{(1)}\left(\Omega_{1}\right)\right] ; \\
Z_{1 n}=n\left[(1-n) H_{n}^{(1)}\left(\Omega_{0}\right)-\Omega_{0} H_{n+1}^{(1)}\left(\Omega_{0}\right)\right] ; \\
Z_{2 n}=\left(a_{n 2}^{1}-\Omega_{1}^{2} / 2\right) H_{n}^{(1)}\left(\Omega_{1}\right)+\Omega_{1} H_{n+1}^{(1)}\left(\Omega_{1}\right) ; \\
d_{1}=\left(1-v_{1}\right) /\left(1-2 v_{1}\right) ; a_{n 2}=n^{2} ; a_{n 2}^{1}=n^{2}-n ; \quad \Omega_{0}=\Omega_{1} L_{1} ; \\
L_{1}=\left(1-2 v_{1}\right) /\left(2\left(1-v_{1}\right)\right) ; \Omega_{1}=\omega a / C_{p 1}
\end{gathered}
$$

With natural oscillations at $r=R_{\infty}$ Shortened Sommerfeld conditions are put in place, i.e.

$$
\lim _{r \rightarrow \infty} \sqrt{r}\left(\frac{\partial \varphi_{1 n}}{\partial r}+i K_{1} \varphi_{1 n}\right)=0, \quad \lim _{r \rightarrow \infty} \sqrt{r}\left(\frac{\partial \varphi_{2 n}}{\partial r}+i K_{2} \varphi_{2 n}\right)=0 .
$$

The solution of the wave equation is sought in the form

$$
\left(\begin{array}{l}
\phi \\
\psi
\end{array}\right)=\sum_{n=0}^{\infty}\left(\begin{array}{l}
\varphi_{1 n}(r) \\
\varphi_{2 n}(r)
\end{array}\right)\left(\begin{array}{c}
\cos \theta \\
\sin \theta
\end{array}\right) \mathrm{e}^{-i \omega t}
$$

where $\omega$-purity; $n$-is the number of waves; $t$-time;

Substituting (3.3) into (3.4), we obtain the Helmholtz equation, whose solution has the form

$$
\left(\begin{array}{l}
\phi \\
\psi
\end{array}\right)=\sum_{n=0}^{\infty}\left(\begin{array}{l}
A_{n} H_{n}^{(1)}\left(K_{1} r\right)+B_{n} H_{n}^{(2)}\left(K_{1} r\right) \\
C_{n} H_{n}^{(1)}\left(K_{2} r\right)+D_{n} H_{n}^{(2)}\left(K_{2} r\right)
\end{array}\right),
$$

where $H_{n}^{(1),(2)}(z)$-Hankel functions of the first and second kind of the n-th order $\alpha=w / c_{p}$ and $\beta=w / C_{S}$-wave numbers; $A_{n}, B_{n}, C_{n}, D_{n}$-arbitrary constants, which are determined from the boundary conditions. From the boundary conditions it follows that $H_{n}^{(2)}(z)$ describes a converging wave; therefore, the solution of (3.5) takes the form

$$
\left(\begin{array}{l}
\phi \\
\psi
\end{array}\right)=\sum_{n=0}^{\infty}\left(\begin{array}{c}
A_{n} H_{n}^{(1)}\left(K_{1} r\right) \\
C_{n} H_{n}^{(1)}\left(K_{2} r\right)
\end{array}\right) .
$$

After setting (3.5) into the boundary conditions (3.3), we obtain a system of algebraic equations with complex coefficients

$$
[D]\{q\}=0,
$$

where $\{q\}=\left\{A_{n}, C_{n}\right\}$-vector column of arbitrary constants; [c] is a square matrix whose elements are expressed in terms of Hankel functions of the first kind of the $\mathrm{n}$-th order. For a system of algebraic equations to have a nontrivial solution it is necessary and sufficient

$$
[c]=0 \text {. }
$$

The roots of the transcendental (3.7) equation describe the frequency of the 
cavity's natural vibrations. The frequency Equation (3.7) takes the following form:

$$
\begin{aligned}
D_{\rho}= & x H_{\rho-1}\left[\left(\rho^{2}-1\right) y H_{\rho-1}(y)-\left(\rho^{3}-\rho+y^{2} / 2\right) H_{\rho}(y)\right] \\
& -H_{\rho}(x)\left[\left(\rho^{3}-\rho+y^{2} / 2\right) y H_{\rho-1}(y)-\left(\rho^{2}+\rho-y^{2} / 4\right) y^{2} H_{\rho}(y)\right],
\end{aligned}
$$

where

$$
x=\omega a(p /(\lambda+2 \mu))^{1 / 2} ; \quad y=\omega a(p / \mu)^{1 / 2}, \lambda \text { and } \mu \text {-Lame coefficients; }
$$
- density of the material. Equation (3.8) after certain transformations can be written in the following form:

$$
\left(\rho^{2}-1\right) F(x) F(y)-\left(y^{2} / 2\right) F(x)+F(y)+\rho^{2}-\left(\rho^{2}-y^{2} / 2\right)^{2}=0,
$$

where $F(x)=x H_{\rho}^{1}(x) / H_{\rho}(x), \rho=1,2,3, \cdots$.

Let us consider in an unbounded medium the radial oscillations of a spherical cavity, accompanied by the emission of longitudinal sound waves, which leads to a loss of energy, and thereby to an attenuation of the oscillations. When $C_{p} \gg C_{S}$ the problem under consideration is equivalent to the problem of the natural oscillations of a spherical body. The roots of the characteristic Equation (3.8) are found by the Mueller method. On the basis of these studies, it is revealed that the mechanical system under consideration has a discrete complex natural frequency. Table 1 shows the results obtained and their comparison, the results of those other authors [13] [14] [15]. The results obtained show that with increasing modulus of elasticity, the corresponding natural frequencies of the mechanical system slowly increase.

Define $\Omega$ for different Poisson coefficients $v_{1}$ and $n$. When $n=0$ we obtain axially symmetric oscillations of the cylindrical hole. The partial Equation (3.8) takes the form

$$
-\Omega d_{1} H_{0}^{(1)}\left(\Omega_{1}\right)+H_{1}^{(1)}\left(\Omega_{1}\right)=0 .
$$

The frequency Equation (3.9) is solved numerically, i.e. the Mueller method. Results of calculations $n \geq 0 \quad\left(v_{1}=0.25\right)$ of the natural oscillations are given in Table 2. As can be seen from the table, the corresponding complex frequencies increase with increasing number of waves along the circumference. Complex frequencies consist of two parts, real $(\operatorname{Re} \Omega)$ and imaginary parts $(\operatorname{Im} \Omega)$ which means natural frequencies and damping coefficients.

Table 1. Comparison of results $(v=0.25)$.

\begin{tabular}{cccc}
\hline № & Our Results & Pao and Mao [14] & Bnron and Parnes [14] \\
\hline 0 & $0.44741-0.44420 \mathrm{i}$ & $0.4464-0.44127 \mathrm{i}$ & $0.4464-0.4410 \mathrm{i}$ \\
1 & $1.09272-0.77653 \mathrm{i}$ & $1.09272-0.7653 \mathrm{i}$ & $1.0929-0.441 \mathrm{i}$ \\
2 & $1.907554-0.89782 \mathrm{i}$ & $1.90754-0.8978 \mathrm{i}$ & $1.9076-0.897 \mathrm{i}$ \\
3 & $2.75652-0.99151$ & $2.75652-0.9915 \mathrm{i}$ & \\
4 & $3.63132-1.06662 \mathrm{i}$ & $3.63132-1.0666 \mathrm{i}$ & \\
5 & $4.52440-1.13140 \mathrm{i}$ & $4.52440-1.1314 \mathrm{i}$ & \\
\hline
\end{tabular}


Table 2. Dependence of the complex Eigen frequencies of a cylindrical hole.

\begin{tabular}{ccccc}
\hline & $n=0$ & $n=1$ & $n=2$ & $n=3$ \\
\hline \multirow{2}{*}{$\Omega_{1}$} & $0.4529 \mathrm{D}+00$ & $0.10927 \mathrm{D}+01$ & $0.19075 \mathrm{D}+01$ & $0.27565 \mathrm{D}+01$ \\
& $-\mathrm{i} 0.47651 \mathrm{D}+00$ & $-\mathrm{i} 0.76538 \mathrm{D}+00$ & $-\mathrm{i} 0.89782 \mathrm{D}+00$ & $-\mathrm{i} 0.99155 \mathrm{D}+00$ \\
$\Omega_{2}$ & & & $0.28621 \mathrm{D}+00$ & $0.72325 \mathrm{D}+01$ \\
$\Omega_{3}$ & & & $-\mathrm{i} 0.17852 \mathrm{D}+00$ & $-\mathrm{i} 0.32283 \mathrm{D}+01$ \\
& & & $0.404607 \mathrm{D}+00$ & $0.12307 \mathrm{D}+00$ \\
& & & $-\mathrm{i} 0.178552 \mathrm{D}+00$ & $-\mathrm{i} 0.22283 \mathrm{D}+00$ \\
\hline
\end{tabular}

The frequency Equations (3.9) depend only on the parameter $v$ (Poisson's ratio). With increasing Poisson's ratio within $0 \leq v \leq 0.4$ The real and imaginary parts of the complex frequency change to $27 \%$. When $v_{1}=0.5$ the medium becomes incompressible, naturally, there are no attenuations. For verification, the results obtained are compared with the results of [10] [16] [17] at $v_{1}=0.25$.

Now let us consider the natural oscillations of a rigid cylindrical inclusion. In this case, we seek the solution of the wave equation and the hard inclusion in the form. On the contact $r=a$ we set the condition for rigid contact. The partial equation for $n=i$ takes the form

$$
\begin{gathered}
\Delta\left(\Omega_{1}\right)=4 \eta H_{1}^{1}\left(\Omega_{1}\right) H_{1}\left(\Omega_{2}\right)-(1-\eta) \Omega_{2} H_{0}\left(\Omega_{2}\right) H_{1}\left(\Omega_{1}\right) \\
-(1+\eta) \Omega_{1} H_{0}\left(\Omega_{1}\right) H_{1}\left(\Omega_{2}\right)+\Omega_{1} \Omega_{2} H_{0}\left(\Omega_{1}\right) H_{0}\left(\Omega_{2}\right) ; \\
\Omega_{1}=\alpha_{1} a ; \Omega_{1}=\beta_{1} a,
\end{gathered}
$$

where $\eta=\rho_{1} / \rho_{2}, \rho_{2}$-tight inclusion density; $\Omega_{1}=\alpha_{1} a ; \Omega_{2}=\beta_{1} a$.

The results of the calculations are presented in Table $3\left(v_{1}=0.25\right)$, according to which $\eta \geq 1$ the real parts of the complex self-purity vanish. When $\eta=0$ we get the vibrations of the environment around the rigid body, i.e. we have only imaginary roots. As a result of using the asymptotic value of the Henkel function (for $a \gg I$ ), we obtained

$$
\omega=-i\left(C_{s 1} / C_{p 1}+1\right) C_{s 1} / a,
$$

The existence of imaginary values of the natural frequency means that oscillatory processes in the system only attenuating. Imaginary Eigen frequencies turns depends on the longitudinal and transverse speed and aperture radius. The existence of a discrete frequency plays an important role for the calculations of underground pipelines are in the ground environment. The obtained numerical results are presented in the form of tables and figures. The appearance of an additional free surface basically thickens and reduces the eigenvalue of the frequency by $10 \%-16 \%$. The existence of natural frequency means that in the vicinity of the free surface of the cylindrical holes may life Rayleigh wave. Thus, according to (3.11), with $n \rightarrow 0$ the real part of the complex frequency does not exist. Now we consider the natural oscillations of a continuous cylindrical inclusion in an elastic medium (Figure 3, Figure 4). The solution of the equation of motion of the medium and the inclusion in the potentials takes the form. At the contact boundary, we set the condition for rigid contact. Solution, substituting in the motion and boundary conditions, we obtain a homogeneous 
Table 3. Comparison of complex frequencies.

\begin{tabular}{cccc}
\hline$n$ & {$[6]$} & Our methodology & {$[9]$} \\
\hline 0 & 0.44647 & 0.45297 & 0.4461 \\
& $-\mathrm{i} 0.44127$ & $-\mathrm{i} 0.47651$ & $-\mathrm{i} 0.4410$ \\
$\mathrm{I}$ & $\mathrm{I} .09272$ & 1.0927 & 0.229 \\
& $-\mathrm{i} 0.7653$ & $-\mathrm{i} 0.76538$ & $-\mathrm{i} 0.441$ \\
2 & $\mathrm{I} .90754$ & $\mathrm{I} .90750$ & $\mathrm{I} .9076$ \\
& $-\mathrm{i} 0.8978$ & $-\mathrm{i} 0.89782$ & $-\mathrm{i} 0.8971$ \\
& 2.75661 & 2.75665 & - \\
\hline
\end{tabular}

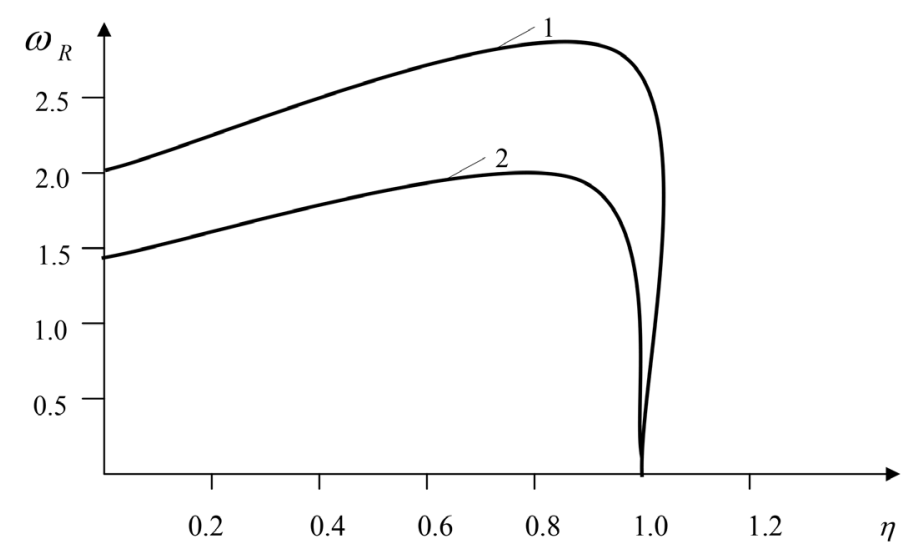

Figure 3. Shows the dependence of the real parts of the natural frequencies on $\eta$.

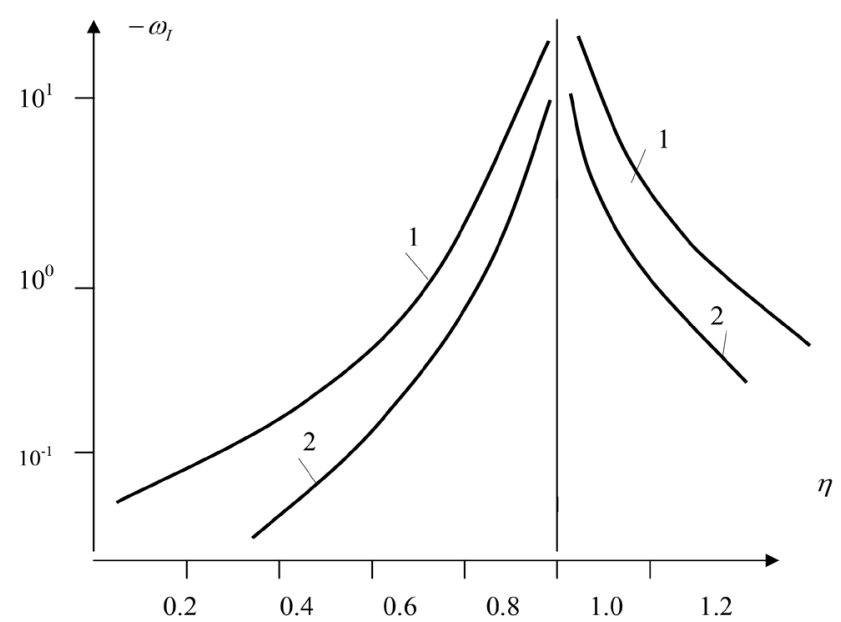

Figure 4. Shows the dependence of the imaginary Eigen frequencies on.

complex algebraic equation in the form. Here the determinant $(C)$ is of the fourth order, its elements have the form

$$
\begin{aligned}
& C_{11}=\left(n^{2}+n-\alpha_{1}^{2} b^{2} / 2\right) H_{n}^{(1)}\left(\alpha_{1} b\right)-\alpha_{1} b H_{n-1}^{(1)}\left(\alpha_{1} b\right) ; \\
& C_{12}=-\bar{\mu}\left[(n+1) H_{n}^{(1)}\left(\beta_{1} b\right)-\beta_{1} b H_{n-1}^{(1)}\left(\beta_{1} b\right)\right] ;
\end{aligned}
$$




$$
\begin{aligned}
& C_{13}=-\bar{\mu} n\left[(n+1) J_{n}\left(\alpha_{2} b\right)-\alpha_{2} b j_{n-1}\left(\alpha_{2} b\right)\right] ; \\
& C_{14}=-\bar{\mu} n\left[(n+1) J_{n}\left(\beta_{2} b\right)-\beta_{2} b J_{n-1}\left(\beta_{2} b\right)\right] ; \\
& C_{21}=n\left[(n+1) H_{n}^{(1)}\left(\alpha_{1} b\right)-\alpha_{1} b H_{n-1}^{(1)}\left(\alpha_{1} b\right)\right] ; \\
& C_{22}=-\left(n^{2}+n-\beta_{1}^{2} b^{2} / 2\right) H_{n}^{(1)}\left(\beta_{1} b\right)+\beta_{1} b H_{n-1}^{(1)}\left(\beta_{1} b\right) ; \\
& C_{23}=-\bar{\mu} n\left[(n+1) J_{n}\left(\alpha_{2} b\right)-\alpha_{2} b J_{n-1}\left(\alpha_{2} b\right)\right] ; \\
& C_{24}=\bar{\mu}\left[\left(n^{2}+n-\beta_{2}^{2} b^{2} / 2\right) J_{n}\left(\beta_{2} b\right)-\beta_{2} b J_{n-1}\left(\beta_{2} b\right)\right] ; \\
& C_{31}=\alpha_{1} b H_{n-1}^{(1)}\left(\alpha_{1} b\right)-n H_{n}^{(1)}\left(\alpha_{1} b\right) ; \\
& C_{32}=-n H_{n}^{(1)}\left(\beta_{1} b\right) ; \\
& C_{33}=-\left[\alpha_{2} b J_{n-1}\left(\alpha_{2} b\right)-n J_{n}\left(\alpha_{2} b\right)\right] ; \\
& C_{34}=-n J_{n}\left(\beta_{1} b\right) ; \\
& C_{41}=-n H_{n}^{(1)}\left(\alpha_{1} b\right) ; \\
& C_{42}=-\beta_{1} b H_{n-1}^{(1)}\left(\beta_{1} b\right)+n H_{n}^{(1)}\left(\beta_{1} b\right) ; \\
& C_{43}=n J_{n}\left(\alpha_{2} b\right) ; \\
& C_{44}=\left[\beta_{2} b J_{n-1}\left(\beta_{2} b\right)-n J_{n}\left(\beta_{2} b\right)\right] ; \\
& \bar{\mu}=\mu_{1} / \mu_{2} .
\end{aligned}
$$

The numerical solution was carried out on a computer with various parameter ratios $\eta=\rho_{1} / \rho_{2} \quad E=E_{1} / E_{2}, \quad v_{1}=v_{2}=0.25$. If the environment is absolutely rigid, then $E_{1} \rightarrow \infty$. (Table 4). The equation corresponding to the condition on the surface loses its meaning, it must be replaced by the boundary condition $\left.U_{r}\right|_{r=b}=\left.U_{\theta}\right|_{r=b}=0$.

Table 4. The change in the complex frequency, depending on $\boldsymbol{E}$ at $\eta=4$; $v_{0}=v=0.14$ (hard contact).

\begin{tabular}{cccccc}
\hline \multicolumn{5}{c}{$E=E_{1} / E_{0}$} \\
\hline & $\Omega$ & 0.4 & 0.6 & 0.8 \\
\hline$\Omega_{1}$ & $\operatorname{Re} \Omega_{1}$ & $2.26428 \mathrm{D}-01$ & $1.8172 \mathrm{D}-01$ & $1.5839 \mathrm{D}-01$ & $1.4324 \mathrm{D}-02$ \\
& $\operatorname{Re} \Omega_{1}$ & $-\mathrm{i} 1.2969 \mathrm{D}-01$ & $-\mathrm{i} 7.7092 \mathrm{D}-02$ & $-\mathrm{i} 5.7656 \mathrm{D}-02$ & $-\mathrm{i} 4.7033 \mathrm{D}-02$ \\
& $\operatorname{Re} \Omega_{2}$ & $3.2339 \mathrm{D}-01$ & $2.3924 \mathrm{D}-01$ & $2.0207 \mathrm{D}-01$ & $1.7995 \mathrm{D}-01$ \\
$\Omega_{2}$ & $\operatorname{Re} \Omega_{2}$ & $-2 \mathrm{i} .5641 \mathrm{D}-01$ & $-\mathrm{i} 2.0152 \mathrm{D}-02$ & $-\mathrm{i} 1.7192 \mathrm{D}-02$ & $-\mathrm{i} 1.5278 \mathrm{D}-01$ \\
& $\operatorname{Re} \Omega_{3}$ & $4.81550 \mathrm{D}+00$ & $4.8144 \mathrm{D}+00$ & $4.8137 \mathrm{D}+00$ & $4.8134 \mathrm{D}+00$ \\
$\Omega_{3}$ & $\operatorname{Re} \Omega_{3}$ & $4.7709 \mathrm{D}+00$ & $-\mathrm{i} 4.7677 \mathrm{D}+00$ & $-\mathrm{i} 4.7667 \mathrm{D}+00$ & $-\mathrm{i} 4.7662 \mathrm{D}-02$ \\
& $\operatorname{Re} \Omega_{4}$ & $4.0795 \mathrm{D}+00$ & $4.0866 \mathrm{D}+00$ & $4.0845 \mathrm{D}+00$ & $4.0831 \mathrm{D}+00$ \\
$\Omega_{4}$ & $\operatorname{Re} \Omega_{4}$ & $5.2295 \mathrm{D}$ & $-\mathrm{i} 5.2428 \mathrm{D}+00$ & $-\mathrm{i} 5.2421 \mathrm{D}+00$ & $-\mathrm{i} 5.2416 \mathrm{D}+00$ \\
& $\operatorname{Re} \Omega_{5}$ & $6.1617 \mathrm{D}+00$ & $6.1612 \mathrm{D}+00$ & $6.1609 \mathrm{D}+00$ & $6.1607 \mathrm{D}+00$ \\
$\Omega_{5}$ & $\operatorname{Re} \Omega_{5}$ & $3.5815 \mathrm{D}+00$ & $-\mathrm{i} 3.5786 \mathrm{D}+00$ & $-\mathrm{i} 3.5781 \mathrm{D}+00$ & $-\mathrm{i} 3.5778 \mathrm{D}-02$ \\
\hline
\end{tabular}


As a result, we obtain a system of algebraic equations with respect to unknowns $A_{n}, B_{n}$. The change in the natural frequencies as a function of $\mathrm{n}$ is shown in Table 5 .

It can be seen that when $\eta=I$ The imaginary parts of the complex frequencies tend to infinity. The picture is similar for a change in $E$, at $E=I$ the imaginary parts of the complex frequencies tend to infinity.

A similar picture was constructed with a change in the parameter E. It can be seen from Figure 3 that when $E=I$ the imaginary parts of the complex frequencies tend to infinity. Let us consider the natural vibrations of cylindrical shells in an infinitely elastic medium. The equation of motion of cylindrical shells and the environment is given in:

$$
\begin{gathered}
\frac{\partial^{2} u}{\partial \theta^{2}}+\frac{\partial W}{\partial \theta}=-\frac{R^{2}}{B} x_{1} \\
\frac{\partial u}{\partial \theta}+b^{2}\left(\frac{\partial^{4} W}{\partial \theta^{4}}+2 \frac{\partial^{2} V}{\partial \theta^{2}}+W\right)+W=\frac{R^{2}}{B} x_{2} \\
\left(\lambda_{o}+2 \mu_{o}\right) \operatorname{graddiv} \boldsymbol{u}-\mu_{0} \text { rotrotu }=\rho_{c} \frac{\partial^{2} u}{\partial t^{2}} \\
\operatorname{grad} \varphi=\frac{\partial \varphi}{\partial r} \boldsymbol{K}_{r}+\frac{1}{r} \frac{\partial \varphi}{\partial \theta} \boldsymbol{K}_{\theta} ; \quad \operatorname{div} \boldsymbol{u}=\frac{1}{r} \frac{\partial\left(r u_{r}\right)}{\partial r}+\frac{1}{r} \frac{\partial u_{\theta}}{\partial \theta} \\
x_{1}=-\left.\sigma_{r \theta}\right|_{r=R}-\rho_{o} h_{o} \frac{\partial^{2} u}{\partial t^{2}} ; \quad x_{2}=-\left.\sigma_{r r}\right|_{r=R}-\rho_{o} h_{o} \frac{\partial^{2} \omega}{\partial t^{2}} \\
b^{2}=\frac{h_{o}^{2}}{12 R^{2}} ; \quad B=\frac{E_{o} h_{o}}{1-v_{o}^{2}} .
\end{gathered}
$$

Here, the index " 0 " refers to the shell, and " $c$ " refers to the environment, $R$ is the radius of the shell, $v_{0}$-Poisson's ratio, $E_{0}$-modulus of elasticity of the shell, $\sigma_{r r}$ and $\sigma_{0 \theta}$-normal and tangential, the components of the reaction from the environment, $\boldsymbol{K}_{r}$ and $\boldsymbol{K}_{\theta}$-unit vectors, $\boldsymbol{u}=u_{r} \boldsymbol{K}_{r}+u_{\theta} \boldsymbol{K}_{\theta}$-vector of displacement of environment, $\lambda_{c}$ and $\mu_{c}$-the Lame coefficients.

The contact between the shell and the environment can be hard or sliding:

$$
\left.U\right|_{r=\alpha}=\left.u_{\theta}\right|_{r=\alpha},\left.W\right|_{r=\alpha}=\left.u_{r}\right|_{r=\alpha}
$$

$$
\left.\eta\left(E_{1}=E_{2}=1, n=2\right) \text { 1) } R=0.5 ; 2\right) R=1 \text {; }
$$

Table 5. Dependence of complex eigen frequencies of hard inclusion on $\eta\left(\rho_{1} / \rho_{2}\right)$.

\begin{tabular}{ccc}
\hline$\eta$ & $R_{e} \Omega_{1}$ & $-i I_{m} \Omega_{1}$ \\
\hline 0.2 & $0.38248 \mathrm{D}+00$ & $0.40845 \mathrm{D}+00$ \\
0.4 & $0.73515 \mathrm{D}+00$ & $0.89541 \mathrm{D}+00$ \\
0.6 & $0.19341 \mathrm{D}+01$ & $0.14480 \mathrm{D}+01$ \\
0.8 & $0.28341 \mathrm{D}+01$ & $0.156907 \mathrm{D}+01$ \\
1.0 & $0.27431 \mathrm{D}-12$ & $0.34807 \mathrm{D}+01$ \\
1.2 & $0.26728 \mathrm{D}-11$ & $0.66809 \mathrm{D}+01$
\end{tabular}


At infinity conditions of "reflecting borders" are put. The solution of equation (3.12) is sought in the form:

$$
\left(\begin{array}{l}
V \\
W \\
u_{\theta} \\
u_{r}
\end{array}\right)=\sum_{n=o}^{\infty}\left(\begin{array}{l}
V_{n}(R) \\
W_{n}(R) \\
U_{\theta}(r) \\
U_{r}(r)
\end{array}\right)\left(\begin{array}{c}
\sin n \theta \\
\cos n \theta \\
\sin n \theta \\
\cos n \theta
\end{array}\right) \cdot \mathrm{e}^{-i \omega t}
$$

where $V_{n}(R), W_{n}(R), U_{\theta}(r)$ and $U_{r}(r)$-displacement amplitude, $\omega=\omega_{R}+i \omega_{I}-$ complex natural frequency

The results of calculations are presented in Table 6.

As an example, consider the axisymmetric vibrations of a cylindrical shell located in an unbounded elastic medium. The differential equation describing the axisymmetric vibrations of a cylindrical shell has the form:

$$
b^{2}\left(\frac{\partial^{n} w}{\partial \theta^{n}}+2 \frac{\partial^{2} w}{\partial \theta^{2}}+w\right)+w=-\frac{\left(1-v_{o}^{2}\right)}{E_{o} h_{o}} R^{2}\left(\rho_{o} h_{o} \frac{\partial^{2} w}{\partial t^{2}}+\sigma_{R R}^{(1)}\right),
$$

where

$$
\begin{gathered}
\sigma_{R R}=\frac{2 \mu}{R^{2}} x_{10} A_{o} \mathrm{e}^{-i w t}, x_{10}=-d_{1} \Omega_{1} H_{0}^{(1)}\left\lfloor\Omega_{1}\right\rfloor+\Omega_{1} H_{1}^{(1)}\left\lfloor\Omega_{1}\right\rfloor, \\
\Omega_{1}=K_{1} R=\frac{\omega}{c_{1}}, \quad d_{1}=\frac{1-v_{1}}{1-2 v_{1}} .
\end{gathered}
$$

Substituting (3.14) into (3.15), we obtain a complex transcendental equation for determining $U_{1}$ :

$$
h=\left(\Omega_{o}^{2} v_{2}-\alpha_{01}\right)+b_{1}-b_{1} d_{1} \Omega_{1} \frac{H_{0}^{(1)}\left(\Omega_{1}\right)}{H_{1}^{(1)}\left(\Omega_{1}\right)},
$$

where

$$
\begin{gathered}
h_{2}=h_{o} / R ; v_{2}=1-v_{o}^{2} ; b_{1}=\frac{E_{1}}{E_{o}} \cdot \frac{1-v_{o}^{2}}{1+v_{1}} ; \alpha_{01}=b^{2}+1 ; \\
d_{1}=\frac{1-v_{1}}{1-2 v_{1}} ; \Omega_{1}=\Omega_{o} \mid S_{1} ; \Omega_{o}=\frac{w}{c_{o}}-R ; c_{o}=\sqrt{E_{o} / \rho_{o}} ; \\
S_{1}=\eta E \cdot \frac{\left(1+v_{1}\right)\left(1-2 v_{1}\right)}{\left(1-v_{1}\right)} ; \eta=\rho_{1} / \rho_{o} ; E=E_{1} / E_{o} .
\end{gathered}
$$

A particular equation for the sliding contact condition takes the form:

$$
\left|\begin{array}{cc}
h_{2} Y_{1 n}-Z_{o}\left(\Omega_{o}\right) X_{1 n} & h_{2} Y_{2 n}-Z_{o}\left(\Omega_{o}\right) Z_{2 n} \\
Z_{1 n} & X_{2 n}
\end{array}\right|=0
$$

Table 6. Dependence of the complex Eigen frequencies of a continuous elastic inclusion on $n$.

\begin{tabular}{ccccc}
\hline$n$ & $m=0$ & $m=1$ & $m=2$ & $m=3$ \\
\hline 0 & 0 & $3.8301 \mathrm{D}+00$ & $7.0223 \mathrm{D}+00$ & $10.1734 \mathrm{D}+00$ \\
1 & $1.8412 \mathrm{D}+00$ & $5.3317 \mathrm{D}+00$ & $8.2401 \mathrm{D}+00$ & $11.7401 \mathrm{D}+00$ \\
\hline
\end{tabular}


where

$$
\begin{gathered}
h_{2}=h_{o} / R ; Y_{1 n}=n H_{n}^{(1)}\left(\Omega_{1}\right)-\Omega_{1} H_{n+1}^{(1)}\left(\Omega_{1}\right) ; \\
Z_{j n}=n\left[(1-n) H_{n}^{(1)}\left(\Omega_{j}\right)+\Omega_{j} H_{n+1}^{(1)}\left(\Omega_{j}\right)\right] ; \\
X_{1 n}=\left(-d_{1} \Omega_{1}^{2}+\alpha_{n 2}^{1}\right) H_{n}^{(1)}\left(\Omega_{1}\right)+\Omega_{1} H_{n+1}^{(1)}\left(\Omega_{1}\right) ; \\
X_{2 n}=\left(\alpha_{2 n}^{1}-\Omega_{2}^{2} / 2\right) H_{n}^{(1)}\left(\Omega_{2}\right)+\Omega_{2} H_{n+1}^{(1)}\left(\Omega_{2}\right) ; \\
Y_{2 n}=n H_{n}^{(1)}\left(\Omega_{2}\right), j=1,2 \\
v_{2}=1-v_{o} ; \alpha_{n 1}=b^{2}\left(n^{2}-1\right)+1 ; \Omega_{1}=\alpha_{1} a ; \\
\Omega_{2}=\beta_{1} \alpha=\Omega_{1}\left(C_{\rho 1} / C_{s 1}\right) ; \Omega_{o}=\alpha_{o} R ; \alpha_{o} a_{0}=\omega / C_{o} ; \\
b^{2}=h^{2} / 12 R^{2} ; b_{1}=E_{1}\left(1-v_{0}^{2}\right) /\left(E_{o}\left(1+v_{1}\right)\right) ; \\
a_{n 2}^{1}=n^{2} ; \beta_{1}=E_{o} h_{o} /\left(1-v_{o}^{2}\right) ; \\
C_{o}=E_{o} / \rho_{o}-\text { wave propagation velocity } \\
Z_{o}\left(\Omega_{o}\right)=b_{1} /\left[\left(\Omega_{0}^{2} v_{2}-a_{n 1}\right)-n^{2} /\left(\Omega_{0}^{2} v_{2}-a_{n 2}\right)\right]
\end{gathered}
$$

In this case we obtain asymmetric vibrations of the cylindrical shell, which are described by equation

$$
h_{2}\left(\Omega_{0}^{2} v_{2}-a_{01}\right)+b_{1}-b_{1} d_{1} \Omega_{1} H_{0}^{(1)}\left(\Omega_{1}\right) / H_{1}^{(1)}\left(\Omega_{1}\right)=0 .
$$

where $\Omega_{1}=\Omega_{o} L_{1} ; L_{1}=\eta E\left(1+v_{1}\right)\left(1-2 v_{1}\right) /\left(1-v_{1}\right)$ (the index " $o$ " corresponds to the shell, and the " 1 " - to the environment).

If we use the asymptotic expression for the Henkel function for $l_{1} \gg 1$, then for the zero and first orders we obtain the expression for complex Eigen frequencies

$$
\Omega_{o}=-i \frac{b_{1} d_{1} l_{1}}{2 h_{2} v_{2}}+\sqrt{\frac{a_{01}}{v_{2}}-\left(\frac{d_{1}}{h_{2} v_{2}}+\left(\frac{d_{1} l_{1} b_{1}}{h_{2} v_{2}}\right)^{2}\right)}
$$

To obtain complex and imaginary Eigen frequencies, it is necessary that condition

$$
\Omega= \begin{cases}\Omega_{R}+i \Omega_{I}, & \left(a_{01} / v_{1}\right)>\left(b_{1} / h_{2} v_{2}\right)+\left(d_{1} l_{1} b_{1} / h_{2} v_{2}\right)^{2} \\ \Omega_{I}, & \left(a_{01} / v_{1}\right)>\left(b_{1} / h_{2} v_{2}\right)+\left(d_{1} l_{1} b_{1} / h_{2} v_{2}\right)^{2}\end{cases}
$$

To satisfy the first condition, the elastic modulus $E$ must satisfy the inequality

$$
E>\left(1+v_{1}\right)\left(b^{2}+1\right) h_{2}^{2}\left(\left[h_{2}+\left(1-v_{1}\right)\right] \eta\left(1-2 v_{1}\right)^{-1}\right)^{-1}\left(1-v_{0}^{2}\right)^{-1}
$$

A similar condition is posed for $\eta$ :

$$
\left.\eta<h_{2}\left(1-2 v_{1}\right)\left(1-v_{1}\right)^{-1}\left[h_{2} a_{01}\left(1+v_{1}\right)\left(1-2 v_{1}\right)^{-1}\right)^{-1}\left(E_{o} / E_{1}\right)-1\right]
$$

Numerical values of asymmetric $x(n=0)$ Eigen frequencies are given in the Table 7.

At $\eta=0.1 ; v_{1}=v_{2}=0.14, h_{o}=0.025$. 
Table 7. Dependence of complex Eigen frequencies of axisymmetric oscillations of cylindrical shells on $E$.

\begin{tabular}{cccccc}
\hline & $E=0.03$ & $E=0.09$ & $E=0.12$ & $E=0.15$ & $E=0.25$ \\
\hline \multirow{2}{*}{$\omega_{R 1}$} & $1.3308 \mathrm{D}-01$ & $2.3976 \mathrm{D}-01$ & $3.2670 \mathrm{D}-01$ & $4.1665 \mathrm{D}-01$ & $1.5270 \mathrm{D}-12$ \\
& $-\mathrm{i} 1.9767 \mathrm{D}-02$ & $-\mathrm{i} 4.5891 \mathrm{D}-02$ & $-\mathrm{i} 6.1776 \mathrm{D}-02$ & $-\mathrm{i} 7.9394 \mathrm{D}-02$ & $-\mathrm{i} 1.3691 \mathrm{D}-01$ \\
\hline
\end{tabular}

As we see, $\left(E_{1} / E_{o}\right) \geq 0.21$ the real parts of the natural frequency vanish, and the behavior of the imaginary parts remains unchanged. The numerical results obtained are confirmed by the condition (3.20). The results of the sliding contact calculations for $n=5$ are given in Table 8 .

If the condition of rigid contact (equality of displacements at $(r=a)$ is put on the interface of the shells with the medium, then the frequency equation (3.20) takes the form

$$
Q_{3 n} Q_{2 n}-Q_{1 n} Q_{4 n}=0
$$

where

$$
\begin{aligned}
& Q_{j n}=\left(\Omega_{0}^{2} v_{2}-n^{2}\right) h^{2} Z_{(2+j) n}+n h_{2} Y_{j n}+b_{1} Z_{j n} ; \\
& Q_{(2+j n) n}=\left(\Omega_{0}^{2} v_{2}-a_{n 1}\right) h_{2} Z_{(j+2) n}-b_{1} X_{j n}+n h_{2} Z_{(j+2) n}, j=1,2 .
\end{aligned}
$$

The results of numerical calculations are given in the Table 9 (with $n=4$, $v_{1}=v_{0}=0.14, \eta=0.3, E=0.2-0.1$ ), according to which for rigid contact imaginary and real parts are $40 \%-60 \%$ larger than when sliding.

Now we consider the case of non-axisymmetric vibrations of a cylindrical layer in an elastic medium. Numerical results were obtained with the following values of the parameters: $E=0.08 ; R=\alpha / b=0.08 ; n=5 ; \eta=0.08-0.72$. The results of calculations are presented in Table 10. When $\eta=I$ we obtain complex natural frequencies of the hole. The change in the complex Eigen frequencies as a function of $\eta$ is shown in Figure 3 and Figure 4 it is seen that as the number of waves along the circle increases, the real and imaginary parts of the complex Eigen frequencies first decrease, and then begin to increase.

From the results (Figure 5) it follows that with decreasing minimum frequencies as a function of $\eta$ are mixed to the right. We consider the natural oscillation of a cylindrical layer of a cylindrical layer in a particular motion in a particular medium in the elastic medium: $[\epsilon]=0$, where

$$
\begin{aligned}
& \mathrm{C}_{11}^{(1)}=\left[n^{2}+n-\frac{\beta^{2} r^{2}}{2}\right] I_{n}\left(\alpha_{k}^{*} r\right)-\alpha_{* r} I_{n-1}^{(1)}\left(\alpha_{*}^{*} r\right) ; \\
& \mathrm{C}_{11}^{(3)}=\left[n^{2}+n-\frac{\beta^{2} r^{2}}{2}\right] H_{n}^{(1)}\left(\alpha_{k}^{*} r\right)-\alpha_{* r} H_{n-1}^{(1)}\left(\alpha_{*}^{*} r\right) ; \\
& \mathrm{C}_{12}^{(3)}=-n(n+1) H_{y}\left(\beta_{k}^{*} r\right)+\beta_{* r} H_{n-1}^{(1)}\left(\beta_{*}^{*} r\right) ; \\
& \mathrm{C}_{41}^{(1)}=-n\left[-(n+1) I_{n}\left(\alpha_{k}^{*} r\right)+\alpha_{* r} I_{n-1}\left(\alpha_{*}^{*} r\right)\right] ; \\
& \mathrm{C}_{41}^{(3)}=-n\left[-(n+1) H_{n}^{1}\left(\alpha_{k}^{*} r\right)+\alpha_{* r} H_{n-1}^{(1)}\left(\alpha_{*}^{*} r\right)\right] ;
\end{aligned}
$$


Table 8. Dependence of the complex frequencies of non-axisymmetric oscillations of cylindrical shells on $E$ with sliding contact.

\begin{tabular}{cccccc}
\hline$\omega$ & $E=0.2$ & $E=0.4$ & $E=0.6$ & $E=0.8$ & $E=1.0$ \\
\hline 1 & $5.9531 \mathrm{D}-02$ & $6.1341 \mathrm{D}-02$ & $6.1901 \mathrm{D}-02$ & $6.2193 \mathrm{D}-02$ & $6.1787 \mathrm{D}-02$ \\
& $-\mathrm{i} 7.5656 \mathrm{D}-02$ & $-\mathrm{i} 7.3121 \mathrm{D}-02$ & $-\mathrm{i} 7.2823 \mathrm{D}-02$ & $-\mathrm{i} 7.1202 \mathrm{D}-02$ & $-\mathrm{i} 6.8760 \mathrm{D}-02$ \\
2 & $1.1582 \mathrm{D}-01$ & $1.1585 \mathrm{D}-01$ & $2.4513 \mathrm{D}-01$ & $4.4340 \mathrm{D}-01$ & $1.1588 \mathrm{D}-01$ \\
& $-\mathrm{i} 6.9000 \mathrm{D}-01$ & $-\mathrm{i} 6.9004 \mathrm{D}-01$ & $-\mathrm{i} 4.4318 \mathrm{D}-01$ & $-\mathrm{i} 6.8910 \mathrm{D}-01$ & $-\mathrm{i} 6.8987 \mathrm{D}-01$ \\
3 & $5.7958 \mathrm{D}+00$ & $5.6652 \mathrm{D}+00$ & $5.7376 \mathrm{D}+00$ & $5.7505 \mathrm{D}+00$ & $5.7971 \mathrm{D}+00$ \\
& $-\mathrm{i} 3.7114 \mathrm{D}+00$ & $-\mathrm{i} 3.6201 \mathrm{D}+00$ & $-\mathrm{i} 3.5791 \mathrm{D}+00$ & $-\mathrm{i} 3.6992 \mathrm{D}+00$ & $-\mathrm{i} 3.7144 \mathrm{D}+00$ \\
4 & $5.4433 \mathrm{D}+00$ & $5.5961 \mathrm{D}+00$ & $5.4244 \mathrm{D}+00$ & $5.0541 \mathrm{D}+00$ & $5.4428 \mathrm{D}+00$ \\
& $-3.8908 \mathrm{D}+00$ & $-\mathrm{i} 3.9481 \mathrm{D}+00$ & $-\mathrm{i} 3.8281 \mathrm{D}+00$ & $-\mathrm{i} 3.9896 \mathrm{D}+00$ & $-\mathrm{i} 3.8914 \mathrm{D}+00$ \\
& $6.8053 \mathrm{D}+00$ & $4.8054 \mathrm{D}+00$ & $6.8055 \mathrm{D}+00$ & $6.8064 \mathrm{D}+00$ & $6.8053 \mathrm{D}+00$ \\
& & & & &
\end{tabular}

Table 9. Dependence of the complex frequencies of non-axisymmetric oscillations of cylindrical shells on $\mathrm{E}$ for rigid contact.

\begin{tabular}{ccccc}
\hline$\omega$ & $E=0.2$ & $E=0.4$ & $E=0.6$ & $E=0.8$ \\
\hline 1 & $2.2642 \mathrm{D}-01$ & $1.8172 \mathrm{D}-01$ & $1.5839 \mathrm{D}-01$ & $1.4324 \mathrm{D}-01$ \\
& $-\mathrm{i} 1.2969 \mathrm{D}-01$ & $-\mathrm{i} 7.7092 \mathrm{D}-02$ & $-\mathrm{i} 5.7656 \mathrm{D}-02$ & $-\mathrm{i} 4.7033 \mathrm{D}-02$ \\
2 & $3.2339 \mathrm{D}-01$ & $3.3921 \mathrm{D}-01$ & $3.0207 \mathrm{D}-01$ & $1.79995 \mathrm{D}-01$ \\
& $-\mathrm{i} 2.5641 \mathrm{D}-01$ & $-\mathrm{i} 2.0152 \mathrm{D}-01$ & $-\mathrm{i} 1.7197 \mathrm{D}-01$ & $\mathrm{i} 1.5278 \mathrm{D}-01$ \\
3 & $4.8155 \mathrm{D}=00$ & $4.8144 \mathrm{D}=00$ & $4.8137 \mathrm{D}+00$ & $4.8134 \mathrm{D}+00$ \\
& $-\mathrm{i} 4.7809 \mathrm{D}+00$ & $-\mathrm{i} 4.7677 \mathrm{D}+00$ & $-\mathrm{i} 4.7667 \mathrm{D}+00$ & $-\mathrm{i} 4.7662 \mathrm{D}+00$ \\
4 & $6.1617 \mathrm{D}+00$ & $6.1612 \mathrm{D}+00$ & $6.1609 \mathrm{D}+00$ & $6.1607 \mathrm{D}+00$ \\
& $-\mathrm{i} 5.5815 \mathrm{D}+00$ & $-\mathrm{i} 5.5786 \mathrm{D}+00$ & $-\mathrm{i} 5.5781 \mathrm{D}+00$ & $-\mathrm{i} 5.5778 \mathrm{D}+00$ \\
\hline
\end{tabular}

Table 10. Dependence of the complex frequencies of a cylindrical layer on $\eta$.

\begin{tabular}{ccccc}
\hline$\omega$ & $\eta=0.08$ & $\eta=0.16$ & $\eta=0.24$ & $\eta=0.32$ \\
\hline 1 & $1.6906 \mathrm{D}-01$ & $4.16999 \mathrm{D}-01$ & $5.3200 \mathrm{D}-01$ & $6.8173 \mathrm{D}-01$ \\
& $-\mathrm{i} 1.5803 \mathrm{D}-02$ & $-\mathrm{i} 3.3343 \mathrm{D}-02$ & $-\mathrm{i} 1.07018 \mathrm{D}-01$ & $-\mathrm{i} 1.0860 \mathrm{D}-01$ \\
2 & $5.1175 \mathrm{D}-13$ & $5.1163 \mathrm{D}-13$ & $5.1139 \mathrm{D}-13$ & $5.1102 \mathrm{D}-13$ \\
& $-\mathrm{i} 9.9134 \mathrm{D}-01$ & $-\mathrm{i} 2.9001 \mathrm{D}+00$ & $-\mathrm{i} 8.8770 \mathrm{D}+00$ & $-\mathrm{j} 1.0389+01$ \\
3 & $6.8166 \mathrm{D}+00$ & $6.8176 \mathrm{D}+00$ & $8.5821 \mathrm{D}+00$ & $8.5830 \mathrm{D}+00$ \\
& $-\mathrm{i} 3.5504 \mathrm{D}+00$ & $-\mathrm{i} 3.6155 \mathrm{D}+00$ & $-\mathrm{i} 6.3848 \mathrm{D}+00$ & $-\mathrm{i} 6.3877+00$ \\
\hline
\end{tabular}

$$
\begin{aligned}
& \mathrm{C}_{42}^{(3)}=\left[n^{2}+n-\frac{\beta^{2} r^{2}}{2}\right] H_{n}^{(1)}\left(\beta_{k}^{*} r\right)+\beta_{* r} H_{n-1}^{(1)}\left(\beta_{*}^{*} r\right) ; \\
& \mathrm{C}_{21}^{(1)}=-\left[n^{2}+n-\frac{\alpha^{2} r^{2}}{2}\right] I_{n}^{(1)}\left(\alpha_{k}^{*} r\right)+\alpha_{* r} H_{n-1}^{(1)}\left(\alpha_{*}^{*} r\right) ; \\
& \mathrm{C}_{12}^{(3)}=-n\left[(n=1) H_{n}^{(1)}\left(\beta_{k}^{*} r\right)-\beta_{* r} H_{n-1}^{(1)}\left(\beta_{*}^{*} r\right)\right] ;
\end{aligned}
$$




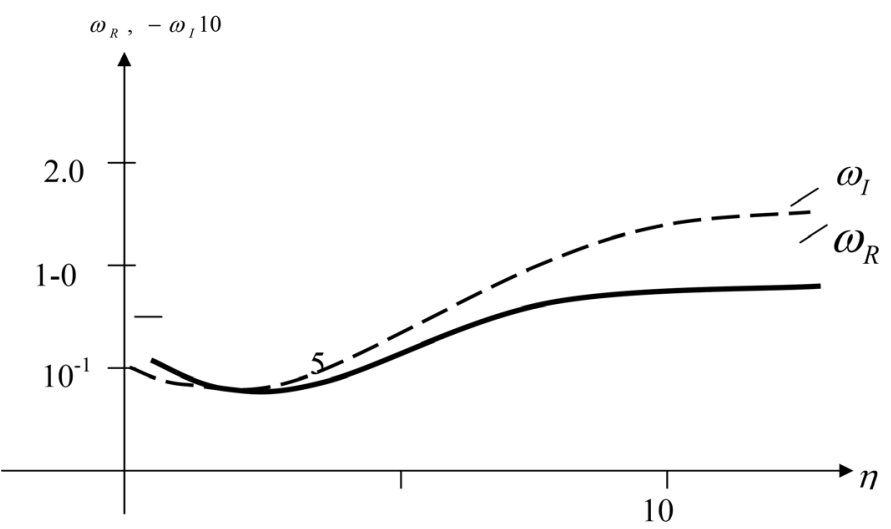

Figure 5. Dependence of natural frequencies $\left(\omega_{R}\right)$ and deformation coefficients $\left(-\omega_{I}\right)$ from $\eta$.

$\alpha^{*}$ and $\beta^{*}$-longitudinal and transverse wave number.

The formulation of the problem of investigating energy dissipation in the propagation of waves in an elastic medium with spherical inclusions is proposed. The coefficient of the so-called scattering coefficient is introduced, which expresses the relationship between the energies of the incident and scattered waves in the sphere. Thus, the scattering coefficient is expressed in terms of the characteristics of the incident and scattered waves.

\section{Oscillations of a Deformable (Elastic or Viscoelastic) Cylinder in a Liquid under the Influence of Internal Pressure}

As an example, let us consider the solution of the problem of oscillations of an elastic hollow cylinder immersed in a liquid under the action of a periodic internal pressure.

In a cylindrical coordinate system $r, \varphi, z$ elastic isotropic cylinder with Lame coefficients $\lambda, \mu$, which can depend on $r$, takes up the volume $R_{1} \leq r \leq R_{2}$, $0 \leq \varphi \leq 2 \pi,-\infty<z<\infty$. Region $r>R_{2}$ fills the ideal density liquid $\rho_{c}$ with the speed of sound waves in it $c_{0}$. On the contact surface of a liquid and a solid $r=R_{2}$ Radial stresses and displacements are assumed to be continuous. On the inner surface $r=R_{1}$ a pressure is established that varies in time according to the harmonic law $p=p_{0} \mathrm{e}^{\mathrm{i} \omega t}$. When solving a stationary problem, it is also necessary to take into account the radiation conditions at infinity. The defining relations connecting the stress tensor $\sigma$ and the strain tensor $\varepsilon$ have the form [13] [15]

$$
\begin{aligned}
& \sigma=\lambda \nabla \cdot u E+2 \mu \varepsilon(u), R_{1} \leq r \leq R_{2} \\
& \sigma=\rho_{c} c_{0}^{2} \nabla \cdot u E, r>R_{2},
\end{aligned}
$$

where $E$ is the unit tensor of the second rank.

When account is taken of the axial symmetry of equation (4.1), we represent it in coordinate form as follows 


$$
\begin{aligned}
& \frac{\partial \sigma_{r r}}{\partial r}+\frac{\sigma_{r r}-\sigma_{\varphi \varphi}}{r}-\rho \frac{\partial^{2} u_{r}}{\partial t^{2}}=0, \\
& l u_{r}=(\lambda+2 \mu) \frac{\partial u_{r}}{\partial r}+\lambda \frac{u_{r}}{r} .
\end{aligned}
$$

In the Equations (4.2) with $r>R_{2}$ should be considered $\lambda=\rho_{c} c_{0}^{2}, \mu=0$. On the inner surface $r=R_{1}$ conditions

$$
\begin{gathered}
\sigma_{r r}=-p_{0} \mathrm{e}^{i \omega t}, \\
\sigma_{r r} /_{r=R_{2}-0}=\sigma_{r r} /_{r=R_{2}+0}, u_{r} /_{r=R_{2}-0}=u_{r} /_{r=R_{2}+0} .
\end{gathered}
$$

The solution is represented in the form $u_{r}=U(r) \mathrm{e}^{\mathrm{i} \omega t}$ and eliminating the stresses in (4.2), we arrive at the equation of stationary oscillations with boundary conditions

$$
\begin{aligned}
& L U+\rho \omega^{2} U=0, L U=\frac{\mathrm{d}}{\mathrm{d} r}\left[(\lambda+2 \mu) \frac{\mathrm{d} U}{\mathrm{~d} r}+\lambda \frac{U}{r}\right]+\frac{2 \mu}{r}\left(\frac{\mathrm{d} U}{\mathrm{~d} r}-\frac{U}{r}\right) \\
& I U\left(R_{1}\right)=-p_{0}, U\left(R_{2}-0\right)=U\left(R_{2}+0\right), l U\left(R_{2}-0\right)=l U\left(R_{2}-0\right) .
\end{aligned}
$$

If we also require that at infinity the function $U$ satisfy the Sommerfeld radiation conditions

$$
\lim _{r \rightarrow \infty} \sqrt{r}|U|=\text { const, } \lim _{r \rightarrow \infty} \sqrt{r}\left(\frac{\mathrm{d} U}{\mathrm{~d} r}+i \frac{\omega}{c_{0}} U\right)=0,
$$

then the boundary value problem (4.4), (4.5) must have a unique solution [14].

For an acoustic environment, the function

$$
U=B_{1} \frac{\mathrm{d}}{\mathrm{d} r} H_{0}^{(2)}\left(\frac{\omega r}{c_{0}}\right),
$$

then the boundary-value problem (4.4) with, $r>R_{2}$ and the radiation conditions (4.5). In (4.6) $B_{1}$ arbitrary constant, $H_{0}^{(2)}-$ Henkel function of the second kind of zero order. Where in

$$
\sigma_{r r}=-\rho_{c} \omega^{2} B_{1} H_{0}^{(2)}\left(\frac{\omega r}{c_{0}}\right) \mathrm{e}^{i \omega t} .
$$

Then, except $B_{1}$ in relations (4.6), (4.7), the problem on a semi-infinite interval $R_{1} \leq r<\infty$ can be reduced to a problem on a finite interval $R_{1} \leq r \leq R_{2}$ for anyone $R_{3} \geq R_{2}$, having determined on the surface $r=R_{3}$ the following boundary condition:

$$
I U=-\rho_{c} \omega^{2} H_{0}^{(2)}\left(\frac{\omega r}{c_{0}}\right)\left[\frac{\mathrm{d} H_{0}^{(2)}}{\mathrm{d} r}\left(\frac{\omega r}{c_{0}}\right)\right]^{-1} U .
$$

In the relation (4.8), the parameter $\omega$ enters meromorphically. For the high-frequency range, using the asymptotic representation of the Henkel function for large arguments

$$
H_{0}^{(2)}(z)=\sqrt{\frac{2}{\pi z}} \mathrm{e}^{-i(z-\pi / 4)}\left[1+O\left(\frac{1}{z}\right)\right] .
$$


Then condition (4.8) can be replaced by the approximate relation

$$
I U\left(R_{3}\right)=-i \rho_{c} \omega c_{0} U\left(R_{3}\right),
$$

with a linearly incoming parameter $\omega$.

Equation (4.9) means, not reflecting the conditions (2.23) for the problems under consideration.

\section{Conclusions}

1) The formulation of the problem is proposed for the natural oscillations of cylindrical bodies in a deformed medium. The task is to find those $\Omega=\Omega_{R}+i \Omega_{i} \quad\left(\Omega_{R}\right.$-real and $\Omega_{i}$-imaginary parts of complex Eigen frequencies) for which the system of equations of motion and the truncated radiation conditions have a solution in the class of infinitely differentiable functions. It is shown that the problem has a discrete spectrum.

2) Two problems of natural oscillations of bodies for an infinite and finite region for some relations of parameters turned out to be equivalent. This is due to the fact that at the outer boundary conditions are set for the absence of reflection.

3) The numerical results obtained for plane mechanical systems in a particular case are compared with known theoretical and experimental values. In short waves $(h / \lambda>0.5)$ the results differ to $10 \%-15 \%$, and in long waves $(h / \lambda>0.5)$ before $25 \%$.

4) From the discussion of the results it is established that with the increase of the elasticity modulus and Poisson's ratio, the corresponding natural frequencies of the mechanical system slowly increase. Natural frequencies $\left(\Omega_{2}\right)$ and damping factors $\left(\Omega_{1}\right)$ at sliding and rigid contact differ up to $15 \%$, and at rigid contact more than $15 \%$. With increasing shell thickness, natural frequencies increase to $10 \%$.

\section{References}

[1] Corn, G. and Korn, T. (1978) Handbook of Mathematics. Science, Moscow, 831 p.

[2] Safarov, I.I. (1992) Oscillations and Waves in Dissipative-Inhomogeneous Media and Structures. Science, Tashkent, $250 \mathrm{p}$.

[3] Safarov, I.I., Akhmedov, M.S. and Boltaev, Z.I. (2015) Distribution of the Natural Waves of Extended Lamellar Viscoelastic Bodies of Variable Thickness. LAP, Lambert Academic Publishing, Saarbrücken, 112 p.

[4] Mayboroda, V.P., Troyanovsky, I.E. and Safarov, I.I. (1983) Free and Forced Oscillations of Systems of Solids on Inhomogeneous Viscoelastic Shock Absorbers. Journal Academy of Sciences, 3, 71-77.

[5] Safarov, I.I., Teshaev, M.X., Akhmedov, M.S. and Boltaev, Z.I. (2017) Properties of Wave Motion in a Cylindrical Shell Is Contact with a Viscous Fluid. Case Studies Journal, 6, 9-35. http://www.casestudiesjournal.com

[6] Safarov, I.I., Teshaev, M.Kh., Boltaev, Z.I. and Nuriddinov, B.Z. (2017) Of Own and Forced Vibrations of Dissipative Inhomogeneous Mechanical Systems. Applied Mathematics, 8, 1001-1015. http://www.scirp.org/journal/am 
https://doi.org/10.4236/am.2017.87078

[7] Safarov, I.I., Teshaev, M.X., Akhmedov, M.Sh. and Boltaev, Z.I. (2017) Distribution Free Waves in Viscoelastic Wedge with and Arbitrary Angle Tops. Applied Mathematics, 8, 736-745. http://www.scirp.org/journal/am

https://doi.org/10.4236/am.2017.85058

[8] Safarov, I.I., Akhmedov, M.Sh. and Boltaev, Z.I. (2015) Setting the Linear Oscillations of Structural Heterogeneity Viscoelastic Lamellar Systems with Point Relations. Applied Mathematics, 6, 225-234. http://www.scirp.org/journal/am https://doi.org/10.4236/am.2015.62022

[9] Safarov, I.I., Akhmedov, M.Sh. and Boltayev, Z.I. (2015) Dissemination Sinusoidal Waves in of a Viscoelastic Strip. Global Journal of Science Frontier Research: F Mathematics \& Decision Sciences, 15, 39-60.

[10] Avliyakulov, N.N. and Safarov, I.I. (2007) Modern Problems of Statics and Dynamics of Underground Turbines. Science, Tashkent, $306 \mathrm{p}$.

[11] Dyakonov, V.M. (1989) Reference Book on Algorithms and Programs. Science, Moscow, $240 \mathrm{p}$.

[12] Bozorov, M.B., Safarov, I.I. and Shokin, Yu.I. (1986) Numerical Simulation of Oscillations of Dissipative Homogeneous and Inhomogeneous Mechanical Systems. Siberian Branch of the Russian Academy of Sciences, Novosibirsk, 188 p.

[13] Safarov, I.I., Teshayev, M.K., Boltayev, Z.I. and Akhmedov, M.Sh. (2017) Damping Properties of Vibrations of Three-Layer VIscoelastic Plate. International Journal of Theoretical and Applied Mathematics, 3,191-198.

http://www.sciencepublishinggroup.com

[14] Pao, Y.H. and Mow, C.C. (1973) The Diffraction of Elastic Waves and Dynamic Stress Concentrations. 694 p.

[15] Safarov, I.I., Akhmedov, M.Sh. and Rajabov, O. (2017) About the Natural Oscillations Viscoelastic Toroidal Shell with the Flowing Fluid. World Wide Journal of Multidisciplinary Research and Development, 3, 295-309. http://www.wwjmrd.com

[16] Safarov, I.I., Akhmedov, M.Sh. and Boltaev, Z.I. (2015) Natural Oscillations of Cylindrical Bodies with External Friction on the Boundary. Applied Mathematics, 6, 629-645. http://www.scirp.org/journal/am https://doi.org/10.4236/am.2015.63057

[17] Safarov, I.I., Kuldashev, N.U. and Ochilov, Sh.B. (2017) Numerical Solution of the Problem of the Impact a Plane Stationary Elastic Waves by a Cylindrical Body. Case Studies Journal, 6, 192-213. http://www.casestudisjournal.com 